\title{
Entropy and Mass Distribution in Disc Galaxies
}

\author{
John Herbert Marr $\mathbb{D}$ \\ Unit of Computational Science, Building 250, Babraham Research Campus, Cambridge CB22 3AT, UK; \\ john.marr@2from.com
}

Received: 18 December 2019; Accepted: 27 January 2020; Published: 8 February 2020

\begin{abstract}
The relaxed motion of stars and gas in galactic discs is well approximated by a rotational velocity that is a function of radial position only, implying that individual components have lost any information about their prior states. Thermodynamically, such an equilibrium state is a microcanonical ensemble with maximum entropy, characterised by a lognormal probability distribution. Assuming this for the surface density distribution yields rotation curves that closely match observational data across a wide range of disc masses and galaxy types and provides a useful tool for modelling the theoretical density distribution in the disc. A universal disc spin parameter emerges from the model, giving a tight virial mass estimator with strong correlation between angular momentum and disc mass, suggesting a mechanism by which the proto-disc developed by dumping excess mass to the core or excess angular momentum to a satellite galaxy. The baryonic-to-dynamic mass ratio for the model approaches unity for high mass galaxies, but is generally $<1$ for low mass discs, and this discrepancy appears to follow a similar relationship to that shown in recent work on the Radial Acceleration Relation (RAR). Although this may support Modified Newtonian Dynamics (MOND) in preference to a Dark Matter (DM) halo, it does not exclude undetected baryonic mass or a gravitational DM component in the disc.
\end{abstract}

Keywords: galaxies: kinematics and dynamics; galaxies: spiral; lognormal density distribution; galaxies: entropy

\section{Introduction}

In classical thermodynamics, entropy is defined as a state function of the system. This is a property that, like volume, density, and internal energy, is dependent only on the current state of the system and independent of how that state was achieved, being in principle measurable to any precision. As with any thermodynamic system, cosmological systems such as a galaxies typically have a large number of particles including gas molecules, dust, and stars, in any of a huge number of possible arrangements within the system defining the amount of information needed to specify the state of the system as a measure of its entropy.

Peebles [1] postulated that galactic spins originated from induced tidal torques from neighbouring structures, whereby initially spherical galaxies developed their pronounced disc shape through acquired angular momentum. The initial spin impulse would have added kinetic energy to the system through induced movement and potential energy through drawing out filaments of stars from merging galaxies. More recently, Herpich et al. [2] proposed that, with the addition of torque, radial migration effectively mixes the angular momentum components of a proto-galaxy to produce the observed circular orbits while conserving total mass and angular momentum, such that the disc's distribution of specific angular momenta $j$ should be near a maximum entropy state.

The stability and ubiquitous nature of galactic discs imply that any relative motion of a displaced star will rapidly be partitioned on the disc rotational time scale. Sellwood and Binney [3] demonstrated that the spiral waves in galaxy discs provide an effective method of radial mixing by churning the baryonic components in a manner that preserves the overall angular momentum with little increase 
in random motion, and on observational grounds, there is generally little tendency for macroscopic turbulence to occur within the stable disc. This does not preclude local gravitational interactions producing other parameters of the velocity distribution, such as the age-velocity dispersion relation described by De Simone et al. [4] in the solar neighbourhood or the formation of local areas of overor under-density such as spiral arms, bars, or voids, and the influence of these on chaotic motion in the solar region was explored by Chakrabarty [5]. There are therefore strong analogies between this additional energy and the addition of a volume of hot gas into a chamber of cold gas with the mixture allowed to diffuse to equilibrium; and the relaxed state of the disc has parallels with adiabatically isolated thermodynamic systems of interacting particles that attain statistical equilibrium with an increase of entropy.

Galactic discs may not be fully isolated as they can eject high-energy stars and may acquire some loose stars and gas from other systems, but once formed, they are thought to have had minimal active development over the past 6 Gyr [6-8]; even the massive Brightest Cluster Galaxies (BCGs) have shown only slow changes in overall brightness over the last 3.5 Gyr of their 10 Gyr of observable history [9], suggesting that external accretion is not required to sustain star formation. Over the recent past, discs may therefore be considered as isolated assemblies of discrete particles undergoing only conservative gravitational interactions, with conservation of independent parameters such as total angular momentum, internal energy, overall number of baryons, and total disc mass. In addition to the conserved macroscopic variables, the observed stable circular orbits imply that all information about the initial conditions has been lost. This implies that reversing the orbits will produce a mirror version of the disc, but will not recreate the original proto-galaxy. On a macro scale, such a system approximates an adiabatic thermodynamic system in thermal equilibrium with a time-invariant, stable mass distribution. This defines a microcanonical ensemble whose entropy is maximized through chaotic mixing of its components as the system approaches equilibrium.

A dynamical approach to describe the relaxation process is difficult as no exact description of the initial state is known, but the similarity and stability of disc galaxies allows them to be considered as idealized relaxed systems in an equilibrium state. The typical disc has $\sim 10^{8}-10^{12}$ stars, and with large $N$, it is necessary to consider the average, statistical properties of the system rather than individual orbits. In contrast to systems with short range repulsive interactions like neutral gases, the attractive long range gravitational force precludes the standard methods of statistical mechanics from being used directly, but the microcanonical distribution can be used to study the statistical properties of any closed system with a fixed total energy $E$; gravitating systems can also be described by this distribution [10]. Such a relaxed state has parallels with adiabatically isolated thermodynamic systems of interacting particles that attain statistical equilibrium with the increase of entropy and an associated mass distribution that is essentially Lognormal (LN). Hence interpreting the disc as a thermodynamic system with high entropy also provides a model universal distribution function for rotationally supported discs with a minimum of free parameters [11,12]. By looking at disc galaxies as isolated systems with maximal entropy, the theoretical dynamic masses can be computed for a wide range of galaxies and compared with their observational masses. We discuss how such systems may have developed their observed mass-density distributions in the disc and compare these with observations to consider potential mechanisms to describe them.

\section{Entropy Changes within an Evolving Galaxy}

Several papers have considered the role of entropy in galaxy formation and structure, and entropy optimization provides a powerful method for data analysis [13]. A maximum entropy approach has been utilized to describe the local structures of the velocity distribution for the phase density function of several samples from the HIPPARCOSand Geneva-Copenhagen survey catalogues [14], while Cubarsi [15] concluded that the entropy method offers an excellent estimation of the truncated velocity distributions of samples containing only thin disc stars. 
Following Peebles' conjecture [1], assume a filament of mass $\delta M$ to be drawn out from a galaxy of total mass $M$. In the absence of torque, the filament will provide an added gravitational potential $\delta E$ to the system, such that the total internal energy $E$ of the system increases. The filament will then fall back into the body of the galaxy such that the potential energy of its individual components will convert to kinetic energy with added radial velocity that will equilibrate over time through mechanisms such as dynamically important strong galactic magnetic fields. These provide the transport of angular momentum required for the collapse of gas clouds and the formation of new stars, drive mass inflow into the centres of galaxies, and can affect the rotation of gas in the outer regions of galaxies, playing important roles in the evolution of galaxies through their direct impact on star formation and stellar feedback-induced turbulence $[16,17]$. This results in a large number of individual stars acquiring additional radial energy, and the volume of the galaxy will expand. The transfer of torque to the galaxy will also transfer kinetic energy of rotation to the system, adding to the total internal energy. The total disc energy (kinetic plus potential) is then the sum of the individual energies:

$$
E=\sum_{i=1}^{N} \epsilon(i)
$$

A system of $N$ particles can be described at any moment by a point in a phase space of $6 N+1$ dimensions, parametrized by the $6 \mathrm{~N}$ canonical co-ordinates and momenta, and time $\left(q_{i}, p_{i}, t\right)$. These $6 \mathrm{~N}$ functions are conserved, and at equilibrium, the statistical behaviour becomes independent of time; therefore, $P(q, p, t) \approx P(q, p)$, with the phase point tracing a one-dimensional curve on a hypersurface of constant $E$ in phase-space [10]. Observations appear to confirm that the equilibrium behaviour of a smoothly rotating galactic disc is independent of its initial conditions [13], obeying statistical regularity in conformity to the microcanonical distribution. The average for any phase-space function $f(p, q)$ is then given by Equation (2):

$$
\langle f(p, q)\rangle=\left(\frac{1}{N ! g(E)}\right) \int f(p, q) \delta(E-H(p, q)) \mathrm{d} p \mathrm{~d} q,
$$

where $g(E)$ is the density of states, $N ! g(E)$ is the volume of phase-space, and $H(p, q)$ is the Hamiltonian [10].

As with all classical systems, because the properties are continuous, the number of microstates is uncountably infinite. The microstates must therefore be grouped by a coarse graining technique by defining their positions and momenta within limited ranges of volume and momenta, $\delta V$ and $\delta p$, to obtain a countable set to define $g(E)$ [18]. For microscopic systems, these limits are set by quantum parameters. For a macroscopic system such as a galactic disc, it is sufficient to define $\delta V$ in terms of a capture volume and $\delta p$ in terms of differential rotational momenta such that individual pairs of stars remain separated over a time scale that is long when measured against their periods of rotation around the disc.

The gravitational potential of a star of one solar mass at a distance of four light years from the Sun is $U \approx-3.78 \times 10^{3} \mathrm{~J} / \mathrm{kg}$, in contrast to that for the Milky Way disc at the position of the Sun $(\approx 8 \mathrm{kpc}$ from the centre) of $U \approx-2.68 \times 10^{11} \mathrm{~J} / \mathrm{kg}$, a factor of $7.1 \times 10^{7}$, and to have a comparable acceleration to the disc, a star of one solar mass would have to enter the Oort cloud ( $\sim 5000$ A.U.). Although a gross simplification, this does justify considering close encounters to be weak interactions. Standard arguments then show that if $N \gg 1$ and the interactions are weak, the relative probability associated with the distribution function $\langle f(p, q)\rangle$ is a distribution of particles in $\mathrm{N}$-dimensional space that is uniform on the manifold. Although the motion of individual stars in a many-body gravitational field is chaotic, with no possibility of recreating the original state of motion of the galaxy at its formation, observations confirm that the equilibrium behaviour of the rotating galactic disc obeys statistical regularity. Because the volume, mass, and internal energy are fixed at equilibrium (the microcanonical ensemble), such a system is one in which all states are equally likely and independent of the initial 
conditions. Maximisation of the entropy is then equivalent to maximising the phase-volume, with the entropy of the system $S(E)$ given by Equation (3):

$$
S(E)=K_{s} \ln (g(E)) .
$$

where $K_{S}$ is a dynamical constant of the system, analogous to $K_{B}$, the Boltzmann constant in thermodynamics.

For the disc system, a more turbulent state may be assumed to have existed at the formation time of the galaxy, when components had acquired angular momentum, but not settled into a regular disc. Such an early structure would have a unique position and momentum signature for each component, and perhaps counter-intuitively, turbulent motion is a more ordered state than laminar flow; hence, the transition towards laminar flow is accompanied by an increase in entropy [19]. Once the disc has stabilized, the gross motion of its components is described by the radial variable, which represents a reduction in information about the system as a whole. An appropriate model for the flow of stars in the galactic disc is suggested by a hydrodynamic analogy to the adiabatic laminar flow of fluid through a thin, flat pipe. Laminar flow is a flow regime characterized by low-momentum convection, but high-momentum diffusion, and these conditions may be extended to the disc components moving in approximately circular orbits at constant linear velocity, with a radial differential velocity across the disc. The stability and ubiquitous nature of galactic discs suggests that any relative motion of a displaced star will rapidly be dissipated, compared with the time scale of disc rotation, to allow it to match the specific momentum of gravitational mass at its new position [2]. Detailed analysis, however, remains complex even within a well-defined hydrodynamic system such as laminar flow through a circular pipe [20].

We seek to maximise entropy using the Wallis probability distribution [21]. Consider a system with $n$ mutually exclusive states, assigned probabilities $\left(p_{1}, p_{2}, \cdots, p_{n}\right)$, and let $q$ quanta of probability, each worth $\delta=1 / q$, be randomly distributed among the $n$ possibilities. Then, $p_{i}=q_{i} / q$, where $p_{i}$ is the probability of the $i$ th position $(i=1,2, \cdots, n)$ and $q_{i}$ is the number of quanta assigned to the $i$ th position, with:

$$
\sum_{i=1}^{n} p_{i}=1,
$$

The probability of $p$ is the multinomial distribution [21]:

$$
\begin{gathered}
\qquad P_{r}(p)=n^{-q} \cdot W, \\
\text { where } W=\frac{q !}{q_{1} ! q_{2} ! \cdots q_{n} !}=\frac{q !}{\left(q p_{1}\right) !\left(q p_{2}\right) ! \cdots\left(q p_{n}\right) !} .
\end{gathered}
$$

The most probable outcome is then the maximum of $W$. We can equally maximise any monotonic increasing function of $\mathrm{W}$, and this is most easily achieved by maximising $q^{-1} \log (W)$, using:

$$
\frac{1}{q} \log W=\frac{1}{q} \log \frac{q !}{\left(q p_{1}\right) !\left(q p_{2}\right) ! \cdots\left(q p_{n}\right) !}
$$

We may simplify (7) by the Stirling approximation [21]. Letting the quanta size $\delta \rightarrow 0$ as the number of quanta $q \rightarrow \infty$, the probability levels go from discrete and grainy to smoothly continuous:

$$
\lim _{q \rightarrow \infty} \frac{1}{q} \log (W) \rightarrow-\sum_{i=1}^{n} p_{i} \log \left(p_{i}\right)
$$

and it may be shown that, for large $n,(8)$ is the entropy of the system [21]:

$$
-\sum_{i=1}^{n} p_{i} \log \left(p_{i}\right)=S\left(p_{1}, p_{2}, \cdots, p_{n}\right) .
$$


Therefore, maximising (8) is equivalent to maximising the entropy [21]. For a normal distribution of $\left(p_{1}, p_{2}, \cdots, p_{n}\right),(8)$ will have a lognormal distribution, which is the distribution of the product of independent random variables [22]. By equating (9) with (3), the lognormal distribution is then the maximum entropy probability distribution for the disc system [23], which is the canonical ensemble, originally derived by Gibbs as the maximum entropy distribution over the classical state space, or phase volume, based on a specified mean value of the energy.

\section{Rationale for a Lognormal Density Distribution}

The ability to build an accurate model is helpful when comparing the theoretical dynamical mass with the observational mass, and a number of methods have been described to generate a disc mass density distribution that mimics any observed Rotational Curve (RC), generally by using ad hoc fitting models. Because the disc is thin compared to its radius, most analytical studies assume it to have negligible thickness and describe it in terms of a pure surface density function, $\Sigma(r)$. This is generally taken to be a function of the surface brightness of the disc, and on empirical grounds, this led to a model of the disc as an exponential function of the form $\Sigma(r)=\Sigma_{0} \exp \left(-r / r_{0}\right)^{1 / n}$ where $r_{0}$ is a characteristic scale factor for the galaxy, with $0.5 \leq n \leq 10$ (the Sérsic function); many simulations of disc formation lead to an approximation of this exponential form with $n=1$ for the galactic disc (the Freeman disc) [2,24].

The unification of thermodynamic concepts of entropy and Shannon's information theory by methods such as Jayne's maximum entropy principle have been well described [25], and a number of papers support the use of a lognormal function on thermodynamic grounds as an appropriate model to describe systems undergoing information loss [2]. Theoretical hydrodynamic simulation by Passot and Vázquez-Semadeni [26] suggested that driven turbulence produces a local LN density distribution, and column density observations of star-forming and non-star-forming molecular clouds have supported this [27-29], with a Probability Distribution Function (PDF) that resembles a lognormal function and a high mass tail attributed to turbulence and self-gravity [30]. Pratt et al. [31] measured the entropy profiles of 31 nearby galaxy clusters selected on X-ray luminosity, without morphological bias. The observed distributions showed a centrally concentrated excess entropy extending to larger radii in lower mass systems, with a large dispersion in scaled entropy in the inner regions, possibly accounted for by cool cores and dynamical activity, but becoming increasingly self-similar at large radii. Pichon [32] used perturbation theory to analyse bi-symmetric instability generated by a structure such as a bar. He derived a distribution function corresponding to the extremum of entropy, given some supplementary constraints such as linearity in the perturbation, and concluded that a state of maximum entropy compatible with total energy and angular momentum conservation corresponded to uniform rotation. Herpich et al. [2] used a radial migration model to generate a state close to maximum entropy. By assuming circularity of the orbits and a maximum entropy distribution of angular momentum, they showed that the derived surface density varied as $\exp \left(R / R_{0}\right)^{1 / 2}$ at large radii and as $R^{-1}$ at small radii for a model with no halo.

In this paper, we used a lognormal function with Newtonian gravity to describe the disc density distribution. This can mimic a wide range of RCs with good accuracy [11] using the general mathematical form modified to the more physical form of Equation (10):

$$
\Sigma(r)=\frac{\Sigma_{0}}{r / r_{\mu}} \exp \left(-\frac{\left[\log \left(r / r_{\mu}\right)\right]^{2}}{2 \sigma^{2}}\right),
$$

where $\Sigma(r)$ is the disc surface density $\left(M_{\odot} \mathrm{kpc}^{-2}\right) ; r$ is the normalized radial variable $(\mathrm{kpc}) ; r_{\mu}$ is the radial scale factor $(\mathrm{kpc}) ; \sigma$ is the standard deviation of the natural logarithm of the radius; and $\Sigma_{0}$ is the surface density of the disc at $r=r_{\mu}\left(M_{\odot} \mathrm{kpc}^{-2}\right)$.

The lognormal model has three independent parameters. $\Sigma_{0}$ only changes the scale of the rotational velocity, $V(r)$, by sliding the curve up or down with no change in shape, but varying $\sigma$ and 
$r_{\mu}$ provides a variety of possible curves that demonstrate typical D-, F-, and R-type curves that are generally consistent with those described by Verheijen [33]. The assumed disc radius, $R_{\max }$, is not a free parameter, as it can always be normalized to unity. Measuring the actual disc radius, however, is non-trivial, as it is often unclear where the disc terminates or if it truncates abruptly. Conventionally, the half-light radius may be used, but for the LN model RCs, $R_{\max }$ was generally defined as the last reported observation, plus one half-bin size, using the quoted values in kpc or converting from arcsecs using the reported distance parameter [12].

The LN distribution fulfils the probability distribution for disc systems in a number of important ways: the radius where the stars orbit must be $>0$; the distribution is highly skewed; and normalization of the function to unity corresponds to the probability that the disc contains all the angular momentum of the galaxy. It is also smoothly asymptotic to zero at the core rather than peaking to a cusp where rotation is unsupported (Equation (10)). The LN function does not describe the bulge, which is assumed to be non-rotating, and the gravitational potential of which is additive to the total potential within the disc. The high angular momentum of the discs and their internal density and velocity profiles resemble hurricanes, and this similarity extends to the null central velocity required for spin as emphasised by Criss and Hofmeister [34].

In contrast to an exponential distribution, these characteristics match the rotation curves for both dwarf galaxies such as NGC 2366 and large galaxies such as M31 (Figures 1a and 2, respectively) and satisfy the observed disc density distributions for many spiral galaxies [35]. Stopping the surface density abruptly at $R_{\max }$ produces a noticeable terminal rise in the RC [11], but although Equation (10) is exact only in the limit $r \rightarrow \infty$, in practice, $\Sigma(r) \rightarrow 0$ as $r \rightarrow R_{\max }$, the maximum radius for observations $(\mathrm{kpc})$ beyond which gas and dust at the galactic periphery become undetectable. Although the terminal density may fall away more gradually, it is difficult to detect this termination observationally because any observable matter will already be included in the disc; matter beyond the detectable disc boundary will by definition be unobserved. Nevertheless, some observers have reported $\mathrm{H}_{\mathrm{I}}$ observations that showed no evidence of stopping at their limit of detection, and with the increasing sensitivity of observations, there is now evidence for some $\mathrm{H}_{\mathrm{I}}$ and molecular gas components extending beyond the original disc boundaries, usually described by adding further exponential components to the disc boundary as a biaxial or triaxial component to the disc [36,37].

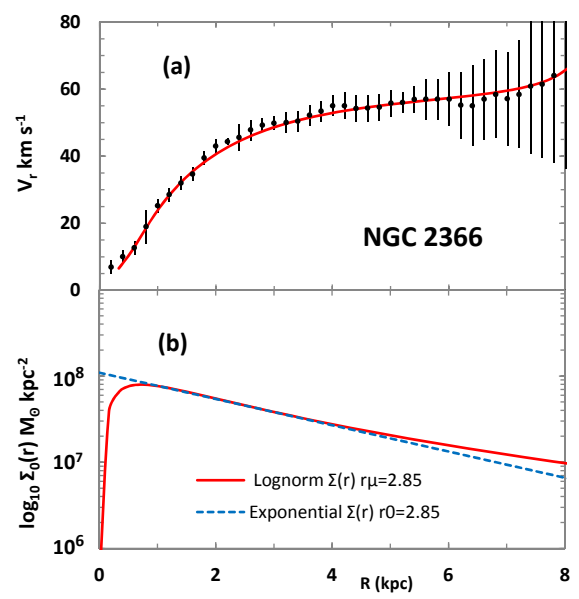

Figure 1. NGC 2366's (a) Rotational velocity for the LN model (solid line) and observations ([38]). (b) Radial distribution of density $\left(M_{\odot} / \mathrm{kpc}^{2}\right)$ for the LN model (solid line) and Freeman exponential disc model (dashed line). 


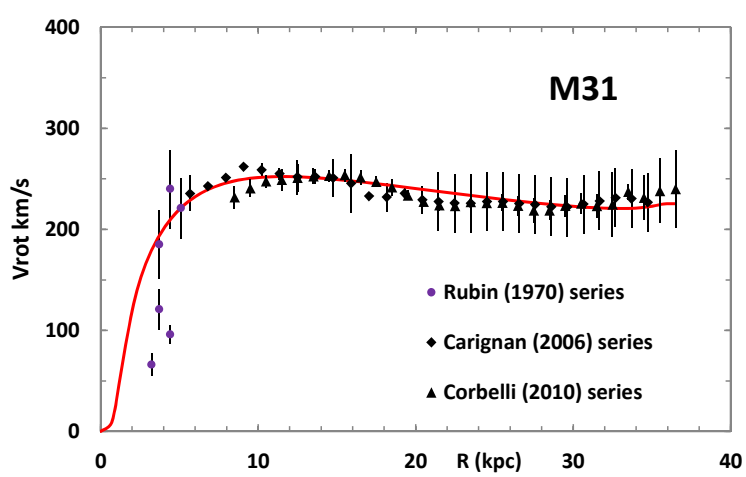

Figure 2. Rotational Curve (RC) for the massive galaxy M31, with rotational velocity for the LN model (solid line) and observations with error bars ([39-41]).

The ability of the LN model to generate realistic RCs with a good fit to observations is demonstrated for the dwarf galaxy NGC 2366 in Figure 1a, with $\Sigma_{0}=1.15 \times 10^{8} M_{\odot} / \mathrm{kpc}^{2}, \sigma=1.16$, $r_{\mu}=2.85 \mathrm{kpc}, R_{\max }=8.3 \mathrm{kpc}$. The high-resolution data were taken from the THINGSsurvey [42]. This survey enabled the effects of random non-circular motions due to collapsing gas clouds in star formation processes, bars, spiral density waves, and warps in the disc to be minimized with the construction of a "bulk" velocity field showing the underlying undisturbed rotation, as described by $\mathrm{Oh}$ et al. [42], with tracers such as $\mathrm{H}_{\mathrm{I}}$ and $\mathrm{H} \alpha$ defined to follow circular orbits. NGC 2366 has a relatively flat F-type curve, but shows a strong terminal rise beyond $7.5 \mathrm{kpc}$. This is a feature of many RCs with an abrupt termination [36], and the fitted LN curve fits the observations well, including the terminal rise.

Figure $1 \mathrm{~b}$ also compares the NGC $2366 \mathrm{LN}$ density curve used to generate the RC with the best fitting exponential density curve. The LN curve (red solid line) is seen to overlie the exponential curve (blue dashed line) for some portion of the radial distance, implying a possible mechanism for the Sérsic exponential models. Observations show there are more stars than expected in a Freeman disc at small radii where bulge stars predominate near the galactic centre, whereas in the LN model, $\Sigma(r) \rightarrow 0$ as $r \rightarrow 0$, reflecting the collapse of the rotation curve where rotation is unsupported and the disc disappears (Figure 1b).

\section{Generating the Spin Parameter and Virial Mass Estimator}

Equation (10) is an integrable function allowing the total disc mass to be calculated (Equation (11)):

$$
M_{\text {disc }}=\Sigma_{0} \sqrt{2 \pi^{3}} r_{\mu}^{2} \sigma \exp \left(\frac{\sigma^{2}}{2}\right)\left[1-\operatorname{erf}\left(\frac{\sigma^{2}-\log \left(R_{\max } / r_{\mu}\right)}{\sigma \sqrt{2}}\right)\right],
$$

and this theoretical disc mass may be compared to observational data for the system. Using a standard function to describe the disc mass distribution also enables other parameters, such as angular momentum and disc total energy, to be computed for a comparative analysis of other galaxy disc properties, such as the dimensionless spin parameter, $\lambda$ (Equation (12)) [1,43-45]:

$$
\lambda \equiv \frac{J|E|^{1 / 2}}{G M^{5 / 2}}
$$

where $M$ is the total gravitational disc mass, $J$ is total angular momentum, and $|E|$ is total energy of the disc, computed using the LN model for a sample of 38 galaxies of varying morphologies using observational RC data, with radii ranging from 3.0-130 kpc and disc masses spanning more than three decades (Figure 3). These gave a mean value of $\lambda \simeq 0.423 \pm 0.014$ [12], comparable to Binney 
and Tremaine's theoretical value for the exponential disc, $\lambda=0.425$ [13], implying that $\lambda$ is a universal function and the LN function is a valid description of the disc as a system of maximum entropy.

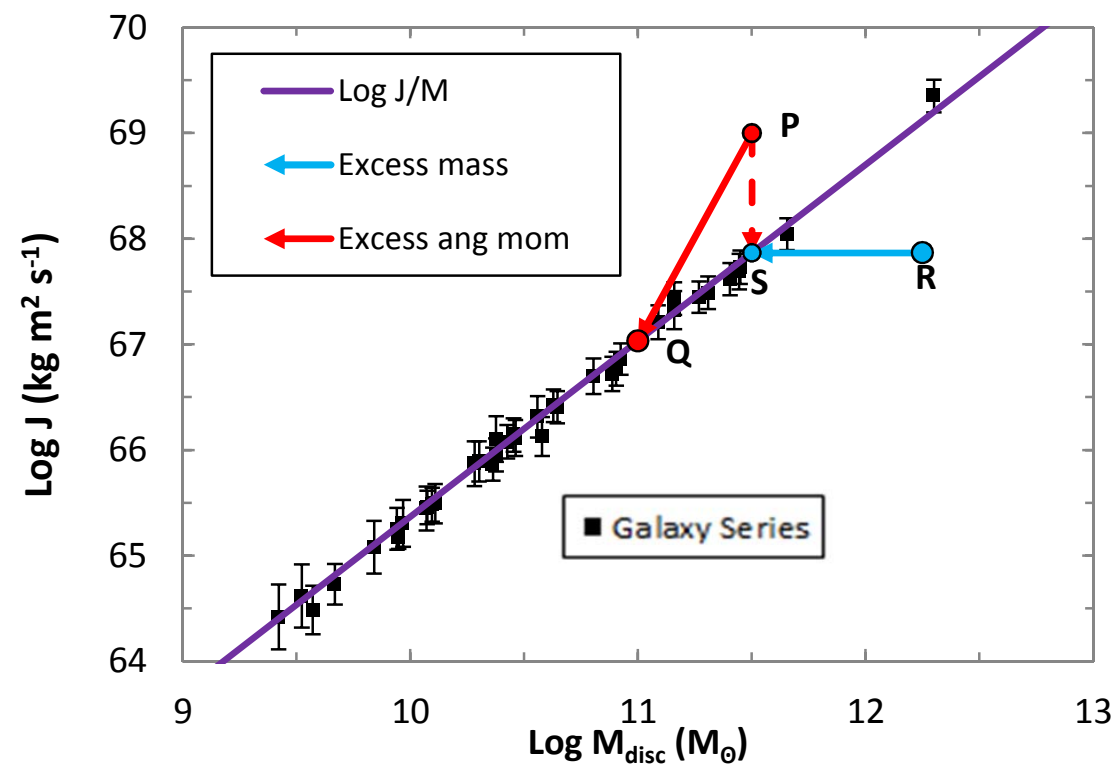

Figure 3. Log-log plot of angular momentum vs. disc mass for the LN model galaxies (squares) and the theoretical slope $5 / 3$ (purple solid line). Idealised evolutionary changes are shown for a disc with excess mass dumping mass into the core while conserving angular momentum (R-S ); and a disc with excess angular momentum shedding mass and angular momentum to a satellite galaxy, leaving a less massive disc (P-Q ).

\section{Changes in Mass and Angular Momentum to Reach Equilibrium}

A $\log$ plot of $J$ vs. $M$ for the LN model, using the derived disc masses, is shown in Figure 3, with the r.m.s. best fitting slope of $1.683 \pm 0.018$, which correlates well with the theoretical slope of $5 / 3$ [46]. It is, however, unlikely that the initial total induced angular momentum/unit mass of the proto-disc would have had this exact spin parameter, and two scenarios are shown: (a) excess $M$ over $J$ (Point $\mathrm{R})$; and (b) excess $J$ over $M$ (Point $P$ ). To reach stability, each of these must move to the $(J / M)$ line as suggested in Figure 3. In Case (a), it is possible that particles with low angular momentum will fall towards the centre of the proto-galaxy by a process of core-dumping; in effect, the proto-disc will lose mass to the bulge (Line R-S). In Case (b), it is unlikely that the proto-disc can lose pure angular momentum (Line P-S), but it may spin-off mass and angular momentum together to move to point $Q$ on the $(J / M)$ line, as shown (Line $\mathrm{P}-\mathrm{Q})$.

Many disc galaxies possess a number of satellite galaxies, and this spin-off mass may go on to form a number of distinct and separate orbiting satellite galaxies, removing both mass and angular momentum from the primary disc. A possible observational consequence of this relationship is that the number of satellite galaxies possessed by a disc system might be inversely proportional to the mass of its bulge. Additional evidence for migration is the exceptionally low metallicities of some nearby molecular clouds compared to the average metallicity of the local interstellar medium and the high metallicity of the Sun [3], suggesting that these clouds formed when the proto-galaxy was forming.

\section{The Mass Discrepancy Relation}

Calculation of the theoretical total dynamic masses $\left(M_{d y n}\right)$ computed with an LN density distribution allows direct comparison with the observational baryonic mass (stars + gas + other components, $M_{b a r}$ ) (Table 1). Total observational disc masses were estimated by summing the components of luminosity mass and computed hydrogen and helium gas mass from published values 
for $\mathrm{H}_{\mathrm{I}}$, although accurate determination of the total baryonic mass distribution remains difficult [47], and even for the Milky Way, there are large intrinsic uncertainties [48,49]. As with all values for $M_{g a s}$ in Table 1, the values for $\mathrm{H}_{\mathrm{I}}$ mass were multiplied by a factor 1.4 to account for the presence of helium, but dust and molecular and ionized gas were not quantified in the mass models, although for neutral gas, this could be derived directly by integrating the $\mathrm{H}_{\mathrm{I}}$ map.

Table 1. Observational masses and computed lognormal masses for 41 disc galaxies.

\begin{tabular}{|c|c|c|c|c|c|c|}
\hline Name & $\begin{array}{c}\mathrm{D} \\
\mathrm{Mpc}\end{array}$ & $\begin{array}{c}\mathbf{M}_{g a s} \\
\left(\log _{10} M_{\odot}\right)\end{array}$ & $\begin{array}{c}M^{*} \\
\left(\log _{10} M_{\odot}\right) \\
\end{array}$ & $\begin{array}{r}\text { Total Mass } \\
\left(\log _{10} M_{\odot}\right)\end{array}$ & $\begin{array}{c}\text { LN Mass } \\
\left(\log _{10} M_{\odot}\right)\end{array}$ & $\begin{array}{l}\text { Refs. } \\
{[\mathrm{D}]\left[M_{\text {disc }}\right]}\end{array}$ \\
\hline DDO 154 & 4.04 & 8.40 & 8.00 & $8.54 \pm 0.27$ & $9.53 \pm 0.15$ & {$[50,51]$} \\
\hline F563-V2 & 61.0 & 9.51 & 9.74 & $9.94 \pm 0.18$ & $10.30 \pm 0.15$ & {$[52,53]$} \\
\hline F568-1 & 85.0 & 9.87 & 9.50 & $10.02 \pm 0.21$ & $10.60 \pm 0.15$ & {$[52,53]$} \\
\hline F568-3 & 77.0 & 9.71 & 9.62 & $9.97 \pm 0.18$ & $10.40 \pm 0.15$ & {$[52,53]$} \\
\hline F568-V1 & 84.8 & 9.53 & 9.82 & $10.00 \pm 0.13$ & $10.60 \pm 0.15$ & {$[50,53]$} \\
\hline F574-1 & 96.0 & 10.32 & 9.52 & $10.38 \pm 0.21$ & $10.40 \pm 0.15$ & {$[52,54]$} \\
\hline IC 2574 & 3.91 & 9.20 & 8.94 & $9.39 \pm 0.18$ & $10.23 \pm 0.20$ & {$[50,53]$} \\
\hline M31 & 0.78 & 9.70 & 11.36 & $11.37 \pm 0.21$ & $11.40 \pm 0.15$ & {$[40,40]$} \\
\hline Malin 1 & 366.0 & 10.97 & --- & $12.00 \pm 0.13$ & $11.94 \pm 0.50$ & {$[55,56]$} \\
\hline Milky Way & - - - & - - & - & $11.83 \pm 0.31$ & $11.40 \pm 0.15$ & {$[-][49]$} \\
\hline NGC 1705 & 5.10 & 8.23 & 8.23 & $8.53 \pm 0.24$ & $9.67 \pm 0.18$ & {$[57,58]$} \\
\hline NGC 2366 & 3.27 & 8.79 & 8.41 & $8.94 \pm 0.21$ & $9.67 \pm 0.20$ & {$[38,50]$} \\
\hline NGC 2403 & 3.16 & 9.67 & 10.04 & $10.20 \pm 0.21$ & $10.80 \pm 0.15$ & {$[50,53]$} \\
\hline NGC 2683 & 8.59 & 8.70 & 10.54 & $10.55 \pm 0.16$ & $10.80 \pm 0.15$ & {$[53,59]$} \\
\hline NGC 2841 & 14.10 & 10.23 & 11.51 & $11.53 \pm 0.18$ & $11.60 \pm 0.15$ & {$[50,53]$} \\
\hline NGC 2903 & 8.90 & 9.49 & 10.74 & $10.76 \pm 0.16$ & $11.20 \pm 0.15$ & {$[50,53]$} \\
\hline NGC 2915 & 3.78 & 8.78 & 7.99 & $8.85 \pm 0.13$ & $10.30 \pm 0.15$ & {$[53,53]$} \\
\hline NGC 2976 & 3.58 & 8.53 & 9.25 & $9.33 \pm 0.18$ & $9.57 \pm 0.15$ & {$[38,50]$} \\
\hline NGC 3198 & 13.80 & 9.80 & 10.36 & $10.47 \pm 0.19$ & $11.10 \pm 0.15$ & {$[50,53]$} \\
\hline NGC 3521 & 8.00 & 9.80 & 10.81 & $10.85 \pm 0.18$ & $11.30 \pm 0.15$ & {$[50,53]$} \\
\hline NGC 3726 & 13.37 & 9.79 & 10.42 & $10.51 \pm 0.17$ & $11.10 \pm 0.15$ & {$[53,59]$} \\
\hline NGC 3741 & 3.0 & 8.45 & 7.24 & $8.48 \pm 0.21$ & $9.42 \pm 0.18$ & {$[53,53]$} \\
\hline NGC 4217 & 20.14 & 9.40 & 10.63 & $10.65 \pm 0.21$ & $10.90 \pm 0.15$ & {$[53,59]$} \\
\hline NGC 4389 & 9.42 & 8.75 & 9.37 & $9.46 \pm 0.16$ & $9.95 \pm 0.10$ & {$[53,59]$} \\
\hline NGC 6946 & 5.5 & 10.43 & 10.43 & $10.73 \pm 0.21$ & $10.90 \pm 0.15$ & {$[53,60]$} \\
\hline NGC 7331 & 13.87 & 10.04 & 11.12 & $11.16 \pm 0.23$ & $11.40 \pm 0.15$ & {$[53,59]$} \\
\hline NGC 7793 & 3.38 & 9.46 & 9.76 & $9.93 \pm 0.22$ & $10.10 \pm 0.16$ & {$[53,59]$} \\
\hline NGC 925 & 8.91 & 10.15 & 10.01 & $10.38 \pm 0.21$ & $10.50 \pm 0.18$ & {$[38,59]$} \\
\hline UGC 128 & 58.5 & 9.96 & 9.76 & $10.17 \pm 0.16$ & $11.10 \pm 0.15$ & {$[50,53]$} \\
\hline UGC 2885 & 75.9 & 10.70 & 11.49 & $11.55 \pm 0.18$ & $12.30 \pm 0.15$ & {$[50,53]$} \\
\hline UGC 5750 & 56.1 & 9.71 & 9.00 & $9.79 \pm 0.21$ & $10.30 \pm 0.15$ & {$[54,54]$} \\
\hline UGC 6399 & 15.5 & 8.85 & 9.32 & $9.44 \pm 0.21$ & $10.10 \pm 0.15$ & {$[61,61]$} \\
\hline UGC 6446 & 15.5 & 9.51 & 9.07 & $9.64 \pm 0.27$ & $10.20 \pm 0.15$ & {$[61,61]$} \\
\hline UGC 6667 & 18.2 & 8.90 & 9.40 & $9.52 \pm 0.13$ & $10.00 \pm 0.18$ & {$[53,59]$} \\
\hline UGC 6818 & 19.5 & 9.00 & 8.60 & $9.15 \pm 0.13$ & $9.84 \pm 0.18$ & {$[53,59]$} \\
\hline UGC 6917 & 15.5 & 9.53 & 9.73 & $9.94 \pm 0.27$ & $10.30 \pm 0.15$ & {$[53,61]$} \\
\hline UGC 6923 & 18.67 & 9.07 & 9.44 & $9.59 \pm 0.21$ & $10.00 \pm 0.15$ & {$[59,62]$} \\
\hline UGC 6969 & 18.6 & 8.79 & 8.49 & $8.97 \pm 0.21$ & $9.94 \pm 0.18$ & {$[63,63]$} \\
\hline UGC 6973 & 36.8 & 9.38 & 10.23 & $10.29 \pm 0.02$ & $10.50 \pm 0.18$ & {$[61,64]$} \\
\hline UGC 6983 & 18.6 & 9.46 & 9.76 & $9.93 \pm 0.24$ & $10.40 \pm 0.15$ & {$[53,65]$} \\
\hline UGC 7089 & 15.5 & 9.40 & 9.28 & $9.65 \pm 0.21$ & $9.97 \pm 0.18$ & {$[61,62]$} \\
\hline
\end{tabular}

The problem of calculating $M^{*}$ is non-trivial, requiring knowledge of the Initial Mass Function (IMF) and the stellar mass-to-light ratio $\left(\mathrm{Y}^{*}\right)$. This itself depends on several poorly constrained factors including age, colour, metallicity, dust extinction, and recent star formation, and Tutukov emphasized how this will change with galactic evolution because $\mathrm{Y}^{*}$ was almost certainly lower in the past when the Star Formation Rate (SFR) was higher and there was less obscuration by dust [6,8]. Unfortunately, these factors are interdependent, giving $\mathrm{Y}^{*}$ a large uncertainty in the mass models, leading many studies 
to assume a min-max disc approach $[38,66]$, with a minimum disc mass from assuming a majority of the rotation arises from the DM halo and the maximum disc hypothesis providing an upper limit on $\mathrm{Y}^{*}$ by maximizing the rotation contribution of the stellar disc. Mass observational errors in Table 1 were taken from quoted values where available or estimated from uncertainties in the $\mathrm{H}_{\mathrm{I}}$ maps and the mass-light ratios such as those cited by McGaugh [50].

The total observational baryonic disc masses $\left(M_{b a r}\right)$ calculated from $M_{g a s}+M^{*}$ for 41 galaxies widely spaced in mass and type are plotted against their corresponding LN dynamic masses $\left(M_{d y n}\right)$ for comparison in Figure 4. The disc masses generated by the LN distribution demonstrated an inverse Mass Deficit Relation (MDR), with an increasing mass deficiency with decreasing disc mass. This confirmed a discrepancy that was described as the Mass Discrepancy Acceleration Relation (MDAR) by Janz et al. [67], and convincingly demonstrated by McGaugh et al. [68] who plotted 2693 points in terms of the local acceleration across the discs of 153 galaxies to show a strong Radial Acceleration Relation (RAR) with a one-fit parameter, the acceleration scale, $g_{+}=1.20 \pm 0.26 \times 10^{-10} \mathrm{~m} \mathrm{~s}^{-2}$ [68], where the mass discrepancy became pronounced. The apparent increase in mass discrepancy with decreasing disc mass in Figure 4 may be correlated with the RAR by a corresponding mass scale, $M_{\dagger}$ (Equation (13)):

$$
M_{\text {dynamic }}=\frac{M_{\text {bar }}}{1-\exp \left(-\sqrt{M_{b a r} / M_{+}}\right)},
$$

This is shown as the dashed line in Figure 4, with an inflexion point at $M_{+}=3.98 \pm 0.54 \times 10^{10} M_{\odot}$, which may be compared to McGaugh's acceleration scale inflection point at $g_{+}$. Despite wide margins of error in deriving the total observational disc mass, the deficiency in observational baryonic mass to computed theoretical dynamic mass appears to bear a systematic inverse relationship to the computed galactic mass, approaching the theoretical disc mass asymptotically for the more massive systems, confirming the earlier observations [68] and lending further support to the LN model as a satisfactory universal predictor of dynamic mass.

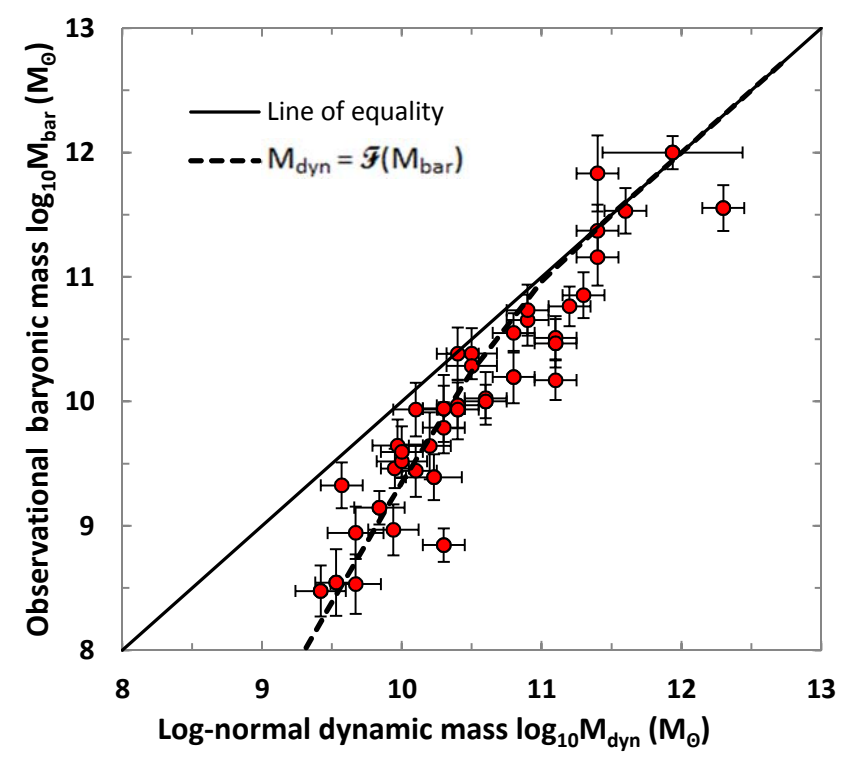

Figure 4. Log-log plot of the modelled lognormal dynamic disc mass vs. the estimated observational baryonic masses $\left(M_{g}+M^{*}\right)$ for a wide range of galaxy masses and types. The solid line is the line of mass equality; the dashed line is for $M_{\text {dynamic }}=f\left(M_{\text {baryonic }}\right)$, with $M_{\dagger}=3.98 \times 10^{10} M_{\odot}$ (see the text). 


\section{Discussion}

The Rotation Curves (RC) of galactic discs provide a vital tool for studying the dynamics of distant galaxies and can be measured with considerable accuracy. Comparing these with the theoretical RCs generated by the total observable mass of the disc and bulge confirmed the discrepancies between them. The failure of observed baryonic mass to account for these RCs led to the concept of a Dark Matter (DM) halo whose properties may be adjusted empirically to fit the observations [69]. However, the inability of experimentalists to identify any DM candidates has led to the postulate that the Newtonian gravitational constant varies at weak field strengths to produce the observed RCs as the Modified Newtonian Dynamics (MOND) hypothesis [70], with gravitational dynamics becoming non-Newtonian in the limit of low acceleration [71-74].

The ability to generate a universal mass model to describe the observed RCs provides a useful method for the analysis of mass distribution in the disc, and a number of methods have been described to generate a universal disc mass density-distribution model that mimics any observed RC [75,76]. Criss and Hofmeister used the virial theorem to model galactic RCs via their linkage of the rotation rate to the gravitational self-potential $(U g)$ and the moment of inertia of oblate spheroids. This allowed galactic mass and volumetric density profiles to be extracted from the velocity and its derivative as functions of equatorial radius, giving a direct, unambiguous, and parameter-free inverse model for rotation curves without DM [34,76]. A computational method was presented by Feng [77] for determining the mass distribution in a mature spiral galaxy from a given rotation curve. Their surface mass density profiles predicted an approximately exponential law of decay, quantitatively consistent with the observed surface brightness distributions, and suggested that Newtonian dynamics can adequately describe the observed rotation behaviour of mature spiral galaxies. similarly, Pavlovich et al. [78] showed that a broad range of galaxy rotation curves could be explained solely by modelling the distribution of baryonic matter in a galaxy.

The maximum entropy model described in this paper provides a physically plausible rationale for a lognormal (LN) surface density distribution that can account for the observed RCs of a wide variety of disc galaxies varying in type, brightness, and mass and generates a reasonable model to establish the dynamic mass of the disc for a wide range of disc masses. It gives a good match to the observational masses of more massive galaxies while approximating the exponential Sérsic distribution over much of the disc radius. The LN model has a universal spin parameter with a highly correlated theoretical mass/angular momentum ratio (Figure 3), suggesting a mechanism by which the disc may stabilize from a proto-disc by dumping excess initial mass to the bulge or shedding excess angular momentum to form a satellite galaxy.

Several independent sources suggest that neither DM nor MOND are universal requirements. Stellar kinematics of elliptical galaxies have suggested there are few unambiguous cases where DM is needed to fit the data, and dynamical modelling of the data indicates the presence of little if any dark matter in these galaxies' halos. [79,80]. Two ultra diffuse galaxies, NGC1052-DF2 and NGC1052-DF4, have very low dispersion velocities nearly identical to the expected values from their stellar masses alone [81,82], although the distance to these is is still under debate with missing mass comparable to other LSB galaxies [83].

The LN model accommodates a scenario in which the missing mass is confined to the disc, and plotting these theoretical dynamic masses of the LN model against the observational masses confirms a Mass Discrepancy Relationship (MDR) that increases with decreasing disc mass, in close agreement with the Radial Acceleration Relation (RAR). McGaugh et al. [68] explained the RAR using MOND, but the presence of DM as a halo or disc component cannot yet be excluded [84], although neither explanation is fully satisfactory on physical grounds, and Janz et al. [67] and Di Cintio and Lelli [85] have suggested that the increase in MDR associated with low mass discs is neither well described by MOND, nor can it arise from a universal NFWprofile, as this would require a mass-dependent DM density profile in $\Lambda \mathrm{CDM}[85]$. 
An alternative possibility may be unobserved baryonic mass in the disc periphery of the faint LSB galaxies. Salem et al. [86] suggested that the Milky Way (MW) hot halo accounts for $4.3 \pm 0.9 \times 10^{10} \mathrm{M} \odot$ or roughly $50 \%$ of these baryons, and others suggested a still larger mass of hot gas [87]. Even for the MW, this hot halo has hitherto been undetectable until recent precise measurements of the movements of satellite galaxies, suggesting that there may be similar undetected hot halos associated with other galaxies and undetected baryonic mass in the LSB galaxies. The Interstellar Medium (ISM) is a mixture of gas and dust remaining from the formation of the galaxy, ejected by stars, and accreted from outside. The gas is very diffuse with some in the form of single neutral atoms, some in the form of simple molecules, and some existing as ions. Its chemical composition is about $91 \%$ hydrogen and $9 \%$ helium. It is observationally important because spectroscopic emission lines from the gas enable measurements of its mass and dynamics, including rotation curves, making it unlikely to be hidden baryonic mass. The total density of dust in the ISM is thought to be considerably less than the gas density, and Draine et al. [88] suggested that $M_{\text {dust }} / M_{(H I+H 2)} \approx 0.01$. The composition of the dust particles is highly variable; grains may vary in size by a factor of $100: 1$, but any excess of dust in LSBs is unlikely to account for hidden mass if its presence in LSB galaxies is in a similar ratio to the MW.

The size of a stellar system without a sharp boundary may be characterized by a gravitational radius, $r_{g}$ [13], and for a star of mass $1 M_{\odot}$, the Oort cloud is thought to extend to approximately $1.5 \times 10^{13} \mathrm{~km}$ if taken to approximate the gravitational sphere of influence $r_{g}$ of a stellar-mass star. The masses of the Oort clouds surrounding such systems are unknown; therefore, we may only estimate possible values from the limited information we have for the Oort cloud of the Solar System. This may contain $10^{11}-10^{12}$ icy bodies, with a total estimated mass of $10^{25}-10^{26} \mathrm{~kg}$ and a mean density $\sim 2 \times 10^{-15} \mathrm{~kg} \mathrm{~km}^{-3}$, although in one estimate, it may approach $2 \%$ of the solar mass, i.e., $\sim 4 \times 10^{28} \mathrm{~kg}$ [89]. The density and mass of the background population of exo-Oort cloud objects is also completely unknown [90], and again with such uncertainty even in the MW, the proportion and mass of icy bodies within other galactic discs is completely unknown; however, they will almost certainly exist. It is a reasonable hypothesis that lower mass galaxies with a higher proportion of gas and lower star formation rates may have a correspondingly high ratio of undetectable icy bodies to detectable baryons, and this proportion may increase with decreasing overall mass.

The LN model cannot exclude MOND or a gravitationally bound DM component as causative of the MDR. However, the good agreement between dynamic and baryonic masses for the RCs of high mass galaxies using a plausible mass-density distribution model suggests that some proportion of the unaccounted-for mass in low mass galaxies may be attributable to uncertainties in mass measurements in the disc peripheries of these Low Surface Brightness (LSB) systems. This may be explained by the presence of DM in the periphery of the disc itself, but the known difficulty and intrinsic errors in assessing the absolute true mass of these systems suggest that at least some of the deficiency may be baryonic [75]. Rather than requiring modification to the law of gravity or a massive undetectable halo of DM, the possibility that it may be accounted for by undetected baryonic matter remains plausible.

Funding: This research received no external funding.

Acknowledgments: I thank the anonymous referees for their thoughtful and constructive reports. I am grateful to Stacy McGaugh for discussions on generating rotation curves, Erwin de Blok and the THINGS team who freely provided their data, and Alexander Tutukov for valuable insights into the properties of the galactic disc.

Conflicts of Interest: The author declares no conflict of interest.

\section{References}

1. Peebles, P.J.E. Origin of the Angular Momentum of Galaxies. Astrophys. J. 1969, 155, 393. [CrossRef]

2. Herpich, J.; Tremaine, S.; Rix, H.W. Galactic disc profiles and a universal angular momentum distribution from statistical physics. Mon. Not. R. Astron. Soc. 2017, 467, 5022-5032, [CrossRef]

3. Sellwood, J.A.; Binney, J.J. Radial mixing in galactic discs. Mon. Not. R. Astron. Soc. 2002, 336, $785-796$. [CrossRef] 
4. De Simone, R.; Wu, X.; Tremaine, S. The stellar velocity distribution in the solar neighbourhood. Mon. Not. R. Astron. Soc. 2004, 350, 627-643. [CrossRef]

5. Chakrabarty, D. Local Phase Space-Shaped by Chaos? Astrophys. Space Sci. Proc. 2009, 8, 151. [CrossRef]

6. Tutukov, A.V.; Shustov, B.M.; Wiebe, D.S. The Stellar Epoch in the Evolution of the Galaxy. Astron. Rep. 2000, 44, 711-718. [CrossRef]

7. Gurovich, S.; Freeman, K.; Jerjen, H.; Staveley-Smith, L.; Puerari, I. The Slope of the Baryonic Tully-Fisher Relation. Astron. J. 2010, 140, 663-676. [CrossRef]

8. Papovich, C.; Labbé, I.; Glazebrook, K.; Quadri, R.; Bekiaris, G.; Dickinson, M.; Finkelstein, S.L.; Fisher, D.; Inami, H.; Livermore, R.C.; et al. Large molecular gas reservoirs in ancestors of Milky Way-mass galaxies nine billion years ago. Nat. Astron. 2016, 1, 0003. [CrossRef]

9. Bellstedt, S.; Lidman, C.; Muzzin, A. The evolution in the stellar mass of brightest cluster galaxies over the past 10 billion years. Mon. Not. R. Astron. Soc. 2016, 460, 2862-2874. [CrossRef]

10. Padmanabhan, T. Statistical mechanics of gravitating systems. Phys. Rep. 1990, 188, 285-362. [CrossRef]

11. Marr, J.H. Galaxy rotation curves with lognormal density distribution. Mon. Not. R. Astron. Soc. 2015, 448, 3229-3241. [CrossRef]

12. Marr, J.H. Angular momentum of disc galaxies with a lognormal density distribution. Mon. Not. R. Astron. Soc. 2015, 453, 2214-2219. [CrossRef]

13. Binney, J.; Tremaine, S. Galactic Dynamics, 2nd ed.; Princeton University Press: Princeton, NJ, USA, 2008.

14. Alcobé, S.; Cubarsi, R. Disk populations from HIPPARCOS kinematic data. Discontinuities in the local velocity distribution. Astron. Astrophys. 2005, 442, 929-946. [CrossRef]

15. Cubarsi, R. Large and small-scale structures of the local Galactic disc. A maximum entropy approach to the stellar velocity distribution. Astron. Astrophys. 2010, 510, A103. [CrossRef]

16. Beck, R. Galactic magnetic fields. Scholarpedia 2007, 2, 2411. [CrossRef]

17. Ntormousi, E. Magnetic fields in massive spirals: The role of feedback and initial conditions. Astron. Astrophys. 2018, 619, L5. [CrossRef]

18. Reif, F. Fundamentals of Statistical and Thermal Physics; Waveland Press: Long Grove, IL, USA, 1965.

19. Sieniutycz, S. Thermodynamic Approaches in Engineering Systems; Elsevier: Amsterdam, The Netherlands, 2016; pp. 260-261.

20. Anand, V.; Nelanti, K. Second Law Analysis of Laminar Flow in a Circular Pipe Immersed in an Isothermal Fluid. J. Thermodyn. 2013, 2013, 234-264. [CrossRef]

21. Jaynes, E. Probability Theory: The Logic of Science; Cambridge University Press: Cambridge, UK, 2003; pp. 351-355.

22. Limpert, E.; Stahel, W.A.; Abbt, M. Log-normal Distributions across the Sciences: Keys and Clues. BioScience 2001, 51, 341-352. [CrossRef]

23. Park, S.Y.; Bera, A.K. Maximum entropy autoregressive conditional heteroskedasticity model. J. Econometr. 2009, 150, 219-230. [CrossRef]

24. Stinson, G.S.; Brook, C.; Macciò, A.V.; Wadsley, J.; Quinn, T.R.; Couchman, H.M.P. Making Galaxies In a Cosmological Context: The need for early stellar feedback. Mon. Not. R. Astron. Soc. 2013, 428, 129-140. [CrossRef]

25. Kapur, J.N.; Kesavan, H.K. Entropy Optimization Principles with Applications; Academic Press: Boston, MA, USA, 1992.

26. Passot, T.; Vázquez-Semadeni, E. Density probability distribution in one-dimensional polytropic gas dynamics. Phys. Rev. E 1998, 58, 4501-4510. [CrossRef]

27. Ostriker, E.C.; Stone, J.M.; Gammie, C.F. Density, Velocity, and Magnetic Field Structure in Turbulent Molecular Cloud Models. Astrophys. J. 2001, 546, 980-1005. [CrossRef]

28. Kainulainen, J.; Beuther, H.; Henning, T.; Plume, R. Probing the evolution of molecular cloud structure. From quiescence to birth. Astron. Astrophys. 2009, 508, L35-L38. [CrossRef]

29. Brunt, C.M.; Federrath, C.; Price, D.J. A method for reconstructing the PDF of a 3D turbulent density field from 2D observations. Mon. Not. R. Astron. Soc. 2010, 405, L56-L60. [CrossRef]

30. Fischera, J. On the probability distribution function of the mass surface density of molecular clouds. II. Astron. Astrophys. 2014, 571, A95. [CrossRef] 
31. Pratt, G.W.; Arnaud, M.; Piffaretti, R.; Böhringer, H.; Ponman, T.J.; Croston, J.H.; Voit, G.M.; Borgani, S.; Bower, R.G. Gas entropy in a representative sample of nearby X-ray galaxy clusters (REXCESS): relationship to gas mass fraction. Astron. Astrophys. 2010, 511, A85. [CrossRef]

32. Pichon, C. Dynamics of Self-Gravitating Disks. Ph.D. Thesis, University of Cambridge, Clare College and Institute of Astronomy, Cambridge, UK, 1994.

33. Verheijen, M.A.W. The Ursa Major Cluster of Galaxies. V. H I Rotation Curve Shapes and the Tully-Fisher Relations. Astrophys. J. 2001, 563, 694-715. [CrossRef]

34. Criss, R.; Hofmeister, A. Galactic Density and Evolution Based on the Virial Theorem, Energy Minimization, and Conservation of Angular Momentum. Galaxies 2018, 6, 115. [CrossRef]

35. Feigelson, E.D.; Babu, G.J. Modern Statistical Methods for Astronomy; Cambridge University Press: Cambridge, UK, 2012.

36. Erwin, P.; Pohlen, M.; Beckman, J.E. The Outer Disks of Early-Type Galaxies. I. Surface-Brightness Profiles of Barred Galaxies. Astron. J. 2008, 135, 20-54. [CrossRef]

37. Herrmann, K.A.; Hunter, D.A.; Elmegreen, B.G. Surface Brightness Profiles of Dwarf Galaxies. I. Profiles and Statistics. Astron. J. 2013, 146, 104. [CrossRef]

38. De Blok, W.J.G.; Walter, F.; Brinks, E.; Trachternach, C.; Oh, S.H.; Kennicutt, R.C., Jr. High-Resolution Rotation Curves and Galaxy Mass Models from THINGS. Astron. J. 2008, 136, 2648-2719. [CrossRef]

39. Rubin, V.C.; Ford, W.K., Jr. Rotation of the Andromeda Nebula from a Spectroscopic Survey of Emission Regions. Astrophys. J. 1970, 159, 379. [CrossRef]

40. Carignan, C.; Chemin, L.; Huchtmeier, W.K.; Lockman, F.J. The Extended H I Rotation Curve and Mass Distribution of M31. Astrophys. J. Lett. 2006, 641, L109-L112. [CrossRef]

41. Corbelli, E.; Lorenzoni, S.; Walterbos, R.; Braun, R.; Thilker, D. A wide-field H I mosaic of Messier 31. II. The disk warp, rotation, and the dark matter halo. Astron. Astrophys. 2010, 511, A89. [CrossRef]

42. Oh, S.H.; de Blok, W.J.G.; Walter, F.; Brinks, E.; Kennicutt, R.C., Jr. High-Resolution Dark Matter Density Profiles of THINGS Dwarf Galaxies: Correcting for Noncircular Motions. Astron. J. 2008, 136, 2761-2781. [CrossRef]

43. Navarro, J.F.; Steinmetz, M. Dark Halo and Disk Galaxy Scaling Laws in Hierarchical Universes. Astrophys. J. 2000, 538, 477-488. [CrossRef]

44. Van den Bosch, F.C. The impact of cooling and feedback on disc galaxies. Mon. Not. R. Astron. Soc. 2002, 332, 456-472. [CrossRef]

45. Teklu, A.F.; Remus, R.S.; Dolag, K.; Beck, A.M.; Burkert, A.; Schmidt, A.S.; Schulze, F.; Steinborn, L.K. Connecting Angular Momentum and Galactic Dynamics: The Complex Interplay between Spin, Mass, and Morphology. Astrophys. J. 2015, 812, 29. [CrossRef]

46. Vettolani, G.; Bergamini, R.; Marano, B.; Zamorani, G. The mass-angular momentum density relation for spiral galaxies. Mon. Not. R. Astron. Soc. 1980, 193, 269-276. [CrossRef]

47. Walter, F.; Brinks, E.; de Blok, W.J.G.; Bigiel, F.; Kennicutt, R.C., Jr.; Thornley, M.D.; Leroy, A. THINGS: The H I Nearby Galaxy Survey. Astron. J. 2008, 136, 2563-2647. [CrossRef]

48. Gnedin, O.Y.; Brown, W.R.; Geller, M.J.; Kenyon, S.J. The Mass Profile of the Galaxy to 80 kpc. Astrophys. J. Lett. 2010, 720, L108-L112. [CrossRef]

49. Bhattacharjee, P.; Chaudhury, S.; Kundu, S. Rotation Curve of the Milky Way out to 200 kpc. Astrophys. J. 2014, 785, 63. [CrossRef]

50. McGaugh, S.S. The Baryonic Tully-Fisher Relation of Gas-rich Galaxies as a Test of $\Lambda$ CDM and MOND. Astron. J. 2012, 143, 40. [CrossRef]

51. Carignan, C.; Purton, C. The "Total" Mass of DDO 154. Astrophys. J. 1998, 506, 125-134. [CrossRef]

52. Swaters, R.A.; Madore, B.F.; Trewhella, M. High-Resolution Rotation Curves of Low Surface Brightness Galaxies. Astrophys. J. Lett. 2000, 531, L107-L110. [CrossRef] [PubMed]

53. McGaugh, S.S. The Baryonic Tully-Fisher Relation of Galaxies with Extended Rotation Curves and the Stellar Mass of Rotating Galaxies. Astrophys. J. 2005, 632, 859-871. [CrossRef]

54. O’Brien, J.G.; Mannheim, P.D. Fitting dwarf galaxy rotation curves with conformal gravity. Mon. Not. R. Astron. Soc. 2012, 421, 1273-1282. [CrossRef]

55. Lelli, F.; Fraternali, F.; Sancisi, R. Structure and dynamics of giant low surface brightness galaxies. Astron. Astrophys. 2010, 516, A11. [CrossRef]

56. Barth, A.J. A Normal Stellar Disk in the Galaxy Malin 1. Bull. Am. Astron. Soc. 2007, 39, 272. [CrossRef] 
57. Elson, E.C.; de Blok, W.J.G.; Kraan-Korteweg, R.C. Star Formation Models for the Dwarf Galaxies NGC 2915 and NGC 1705. Astron. J. 2012, 143, 1. [CrossRef]

58. Romano, D.; Tosi, M.; Matteucci, F. Formation and evolution of late-type dwarf galaxies-I. NGC1705 and NGC1569. Mon. Not. R. Astron. Soc. 2006, 365, 759-778. [CrossRef]

59. Tully, R.B.; Courtois, H.M.; Dolphin, A.E.; Fisher, J.R.; Héraudeau, P.; Jacobs, B.A.; Karachentsev, I.D.; Makarov, D.; Makarova, L.; Mitronova, S.; et al. Cosmicflows-2: The Data. Astron. J. 2013, 146, 86. [CrossRef]

60. Smith, B.J.; Struck, C.; Hancock, M.; Appleton, P.N.; Charmandaris, V.; Reach, W.T. The Spitzer Spirals, Bridges, and Tails Interacting Galaxy Survey: Interaction-Induced Star Formation in the Mid-Infrared. Astron. J. 2007, 133, 791-817. [CrossRef]

61. Sanders, R.H.; Verheijen, M.A.W. Rotation Curves of Ursa Major Galaxies in the Context of Modified Newtonian Dynamics. Astrophys. J. 1998, 503, 97-108. [CrossRef]

62. Zavala, J.; Avila-Reese, V.; Hernández-Toledo, H.; Firmani, C. The luminous and dark matter content of disk galaxies. Astron. Astrophys. 2003, 412, 633-650. [CrossRef]

63. Bottema, R. Dark and luminous matter in the NGC 3992 group of galaxies. II. The dwarf companions UGC 6923, UGC 6940, UGC 6969, and the Tully-Fisher relation. Astron. Astrophys. 2002, 388, 809-825. [CrossRef]

64. Cappellari, M.; Emsellem, E.; Krajnović, D. The ATLAS ${ }^{3 D}$ project-I. A volume-limited sample of 260 nearby early-type galaxies: science goals and selection criteria. Mon. Not. R. Astron. Soc. 2011, 413, 813-836. [CrossRef]

65. Stark, D.V.; McGaugh, S.S.; Swaters, R.A. A First Attempt to Calibrate the Baryonic Tully-Fisher Relation with Gas-Dominated Galaxies. Astron. J. 2009, 138, 392-401. [CrossRef]

66. Van Albada, T.S.; Sancisi, R. Dark Matter in Spiral Galaxies. Phil. Trans. R. Soc. Lond. A 1986, 320, 447-464.

67. Janz, J.; Cappellari, M.; Romanowsky, A.J.; Ciotti, L.; Alabi, A.; Forbes, D.A. The mass discrepancy acceleration relation in early-type galaxies: Extended mass profiles and the phantom menace to MOND. Mon. Not. R. Astron. Soc. 2016, 461, 2367-2373. [CrossRef]

68. McGaugh, S.S.; Lelli, F.; Schombert, J.M. Radial Acceleration Relation in Rotationally Supported Galaxies. Phys. Rev. Lett. 2016, 117, 201101. [CrossRef] [PubMed]

69. Casertano, S.; van Gorkom, J.H. Declining rotation curves-The end of a conspiracy? Astron. J. 1991, 101, 1231-1241. [CrossRef]

70. Milgrom, M. A modification of the Newtonian dynamics as a possible alternative to the hidden mass hypothesis. Astrophys. J. 1983, 270, 365-370. [CrossRef]

71. Sanders, R.H. The Published Extended Rotation Curves of Spiral Galaxies: Confrontation with Modified Dynamics. Astrophys. J. 1996, 473, 117. [CrossRef]

72. De Blok, W.J.G.; McGaugh, S.S. Testing Modified Newtonian Dynamics with Low Surface Brightness Galaxies: Rotation Curve FITS. Astrophys. J. 1998, 508, 132-140. [CrossRef]

73. Sanders, R.H.; McGaugh, S.S. Modified Newtonian Dynamics as an Alternative to Dark Matter. Annu. Rev. Astron. Astrophys. 2002, 40, 263-317. [CrossRef]

74. Swaters, R.A.; Sanders, R.H.; McGaugh, S.S. Testing Modified Newtonian Dynamics with Rotation Curves of Dwarf and Low Surface Brightness Galaxies. Astrophys. J. 2010, 718, 380-391. [CrossRef]

75. Christodoulou, D.M.; Kazanas, D. The Case against Dark Matter and Modified Gravity: Flat Rotation Curves Are a Rigorous Requirement in Rotating Self-Gravitating Newtonian Gaseous Discs. J. Mod. Phys. 2016, 7, 680-698. [CrossRef]

76. Hofmeister, A.M.; Criss, R.E.; Criss, E.M. Verified solutions for the gravitational attraction to an oblate spheroid: Implications for planet mass and satellite orbits. Planet. Space Sci. 2018, 152, 68-81. [CrossRef]

77. Feng, J.; Gallo, C. Mass Distribution in Rotating Thin-Disk Galaxies According to Newtonian Dynamics. Galaxies 2014, 2, 199-222. [CrossRef]

78. Pavlovich, K.; Pavlovich, A.; Sipols, A. Newtonian explanation of galaxy rotation curves based on distribution of baryonic matter. arXiv 2014, arXiv:1406.2401.

79. Kronawitter, A.; Saglia, R.P.; Gerhard, O.; Bender, R. Orbital structure and mass distribution in elliptical galaxies. Astron. Astrophys. Suppl. Ser. 2000, 144, 53-84. [CrossRef]

80. Bosma, A. Dark Matter in Galaxies: Observational overview. Symp.-Int. Astron. Union 2004, $220,39$. [CrossRef]

81. Danieli, S.; van Dokkum, P.; Conroy, C.; Abraham, R.; Romanowsky, A.J. Still Missing Dark Matter: KCWI High-resolution Stellar Kinematics of NGC1052-DF2. Astrophys. J. Lett. 2019, 874, L12. [CrossRef] 
82. Van Dokkum, P.; Danieli, S.; Abraham, R.; Conroy, C.; Romanowsky, A.J. A Second Galaxy Missing Dark Matter in the NGC 1052 Group. Astrophys. J. Lett. 2019, 874, L5. [CrossRef]

83. Trujillo, I.; Beasley, M.A.; Borlaff, A.; Carrasco, E.R.; Di Cintio, A.; Filho, M.; Monelli, M.; Montes, M.; Román, J.; Ruiz-Lara, T.; et al. A distance of 13 Mpc resolves the claimed anomalies of the galaxy lacking dark matter. Mon. Not. R. Astron. Soc. 2019, 486, 1192-1219. [CrossRef]

84. Navarro, J.F.; Benítez-Llambay, A.; Fattahi, A.; Frenk, C.S.; Ludlow, A.D.; Oman, K.A.; Schaller, M.; Theuns, T. The origin of the mass discrepancy-acceleration relation in $\Lambda$ CDM. Mon. Not. R. Astron. Soc. 2017, 471, 1841-1848. [CrossRef]

85. Di Cintio, A.; Lelli, F. The mass discrepancy acceleration relation in a $\Lambda$ CDM context. Mon. Not. R. Astron. Soc. 2016, 456, L127-L131. [CrossRef]

86. Salem, M.; Besla, G.; Bryan, G.; Putman, M.; van der Marel, R.P.; Tonnesen, S. Ram pressure stripping of the large magellanic cloud's disk as a probe of the milky way's circumgalactic medium Astrophys. J. 2015, 815, 77. [CrossRef]

87. Gupta, A.; Mathur, S.; Krongold, Y.; Nicastro, F.; Galeazzi, M. A Huge Reservoir of Ionized Gas around the Milky Way: Accounting for the Missing Mass? Astrophys. J. Lett. 2012, 756, L8. [CrossRef]

88. Draine, B.T.; Dale, D.A.; Bendo, G. Dust Masses, PAH Abundances, and Starlight Intensities in the SINGS Galaxy Sample. Astrophys. J. 2007, 663, 866-894. [CrossRef]

89. Mendis, D.A.; Marconi, M.L. A note on the total mass of comets in the solar system. Earth Moon Planets 1986, 36, 187-190. [CrossRef]

90. Moro-Martín, A. Origin of 'Oumuamua. I. An Ejected Protoplanetary Disk Object? Astrophys. J. 2018, 866, 131. [CrossRef]

(C) 2020 by the authors. Licensee MDPI, Basel, Switzerland. This article is an open access article distributed under the terms and conditions of the Creative Commons Attribution (CC BY) license (http:/ / creativecommons.org/licenses/by/4.0/). 